\title{
Curvas de crescimento para codornas de corte
}

\author{
Growth curve for quails
}

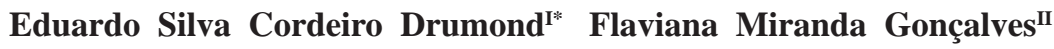 \\ Rogério de Carvalho Veloso ${ }^{\mathrm{I}}$ Jéssica Miranda AmaralIII Lucília Valadares Balotin ${ }^{\mathrm{III}}$ \\ Aldrin Vieira Pires ${ }^{\mathrm{IV}}$ Joerley Moreira ${ }^{\mathrm{IV}}$
}

\section{RESUMO}

\begin{abstract}
Objetivou-se com este trabalho comparar modelos não lineares para descrever o crescimento de codornas de corte machos e fêmeas do nascimento ao 42 - dia de idade. Foram utilizados 24.679 registros de peso corporal de codornas de corte da linha fêmea LF1, pertencente ao Programa de Melhoramento de Codornas da UFVJM, em Diamantina, MG. As codornas de três gerações sucessivas foram pesadas a cada sete dias (nascimento, 7, 14, 21, 35 e 42 dias de idade). Os modelos de Brody, von Bertalanffy, Richards, Logístico e Gompertz foram ajustados ao conjunto de dados observados. Os parâmetros dos modelos foram estimados, para machos e fêmeas separadamente, pelo algoritmo de Gauss Newton, utilizando-se o procedimento NLIN do SAS. Os critérios utilizados para escolha do modelo de melhor ajuste da curva de crescimento foram o coeficiente de determinação $\left(R^{2}\right)$, o desvio padrão assintótico (DPA), o desvio médio absoluto dos resíduos (DMA) e o índice assintótico (IA). Dos cinco modelos utilizados, apenas o modelo de Richards não apresentou convergência, tanto para machos como para fêmeas. O menor valor para o índice assintótico (IA) foi observado para o modelo de Gompertz em machos $(26,57)$ e o Logístico para fêmeas $(25,02)$, indicando serem estes os modelos que mais se ajustaram aos dados.
\end{abstract}

Palavras-chave: Coturnix coturnix coturnix, curva de crescimento, modelos não lineares.

\section{ABSTRACT}

The objective of this study was comparing nonlinear models to describe the growth of quails males and females from birth to 42 days old. We used 24,679 records of body weight of quails from the female line LF1, belonging to UFVJM's Improvement Program of Quail in Diamantina, MG. The three successive generations of quails were weighed every seven days (birth, 7, 14, 21, 35 and 42 days old). The models Brody, von Bertalanffy, Richards, Logistic and Gompertz were fitted to the data set. The model parameters were estimated for males and females separately, the Gauss Newton algorithm using the NLIN procedure of SAS The criteria used to choose the model best fit the curve of growth were the coefficient of determination $\left(R^{2}\right)$, the asymptotic standard deviation (ASD), the mean absolute deviation of the waste (DMA) and the asymptotic index (AI). Of the five models used only the Richards model showed no convergence, both for males and females. The lower asymptotic value for the index (AI) was observed for the Gompertz model in males (26.57) for females and Logistics (25.02), indicating that these models that best fit the data.

Key words: Coturnix coturnix coturnix, growth curve, non-linear models.

\section{INTRODUÇÃO}

Em produção animal, é comum o uso de modelos matemáticos para descrever funções biológicas, como o crescimento, o que tem se mostrado bastante útil, principalmente nas pesquisas de melhoramento genético. Assim, variáveis quantitativas são tomadas para representar fatores que influenciam o fenômeno (RONDON et al., 2002).

A propriedade essencial das funções de crescimento é que a informação contida em um número de pontos, dados pelas coordenadas do peso e tempo, pode ser sumarizada em poucos parâmetros

'Programa de Pós-graduação em Zootecnia (PPGZOO), Universidade Federal dos Vales do Jequitinhonha e Mucuri (UFVJM), Rodovia MGT, 367, Km 583, n. 5000, Alto da Jacuba, 39100-000, Diamantina, MG, Brasil. E-mail: eduardodrumond@yahoo.com.br. *Autor para correspondência.

"Mestre em Zootecnia, PPGZOO, UFVJM, Diamantina, MG, Brasil.

IIICurso de Zootecnia, Departamento de Zootecnia (DZO), UFVJM, Diamantina, MG, Brasil.

${ }^{\mathrm{IV}}$ DZO, UFVJM, Diamantina, MG, Brasil. 
com interpretação biológica, fornecendo estimativas de taxas de crescimento, grau de maturidade e tamanho à maturidade (LAIRD \& HOWARD, 1967). Esses parâmetros possibilitam detectar, em uma população, animais mais pesados em idades mais precoces (THOLON \& QUEIROZ, 2009) e fornecem informações importantes sobre a variação genética e ambiental que ocorre entre as avaliações consecutivas (MANSOUR et al., 1991).

Sendo assim, o estudo da curva de crescimento possibilita uma melhor avaliação da população, permitindo planejar mudanças na curva de crescimento dos animais, por meio de seleção e otimização das estratégias de alimentação (GOUS, 1998), priorizando as necessidades nutricionais em cada fase de crescimento.

Linhagens diferentes respondem diferentemente ao processo de seleção e cruzamentos entre as linhagens poderiam alterar a curva de crescimento das aves, obtendo-se ganhos com heterose, proveniente das divergências entre as linhagens. Portanto, segundo KNIZETOVA et al. (1991), o conhecimento dos parâmetros da curva de crescimento de diferentes raças ou linhas se torna essencial para a formação de programas de melhoramento, cujo objetivo seja produzir aves híbridas do tipo carne, com rápido crescimento inicial e linhas parentais com baixo peso final, aliado à seleção para mudar a forma da curva de crescimento.

Objetivou-se com este trabalho comparar modelos não lineares para descrever o crescimento de codornas de corte machos e fêmeas da linha LF1 do nascimento ao $42^{\circ}$ dia de idade.

\section{MATERIAL E MÉTODOS}

O presente estudo foi realizado nas instalações do Programa de Melhoramento Genético de Codornas da Universidade Federal dos Vales do Jequitinhonha e Mucuri (UFVJM), em Diamantina, MG.

Foram utilizados 24.679 registros de peso corporal de codornas de corte da linha fêmea LF1, em três gerações sucessivas, pesadas a cada sete dias, ou seja, ao nascimento, aos 7, 14, 21, 35 e 42 dias de idade. Para as análises, foram utilizadas codornas com no mínimo três registros de peso, coletados no período de junho de 2010 a maio de 2011.

As aves foram criadas em piso com cama de maravalha, com aquecimento artificial através de lâmpadas infravermelho de 250w até os 21 dias de idade, com temperatura ambiente em torno de 32 a $35^{\circ} \mathrm{C}$. As codornas receberam água e ração à vontade durante todo o período experimental, sendo que a ração continha $25 \%$ PB e $2900 \mathrm{kcal}$ de $\mathrm{EM} \mathrm{kg}{ }^{-1}$, do nascimento ao $21^{\circ}$ dia de idade, e $24 \%$ PB e $2925 \mathrm{kcal}$ de $\mathrm{EM} \mathrm{kg}^{-1}$, do $22^{\circ}$ ao $42^{\circ}$ dia de idade, segundo informações de exigências nutricionais da literatura nacional (OLIVEIRA et al., 2002; CORRÊA et al., 2007; VELOSO et al., 2012).

Ao nascimento, as aves foram identificadas individualmente, pesadas e transferidas para os boxes. No $35^{\circ}$ dia de idade, foi realizada a seleção para peso corporal de 210 fêmeas e 105 machos, que constituíram a geração seguinte e assim sucessivamente.

$\mathrm{Na}$ primeira geração, foram realizadas três eclosões com um total de 793 aves, na segunda geração, cinco eclosões, com um total de 2.189 aves, e, na terceira geração, quatro eclosões, totalizando 1.528 aves. As eclosões de uma mesma geração foram realizadas a cada sete dias, sendo que todas as codornas receberam as mesmas práticas de manejo, conforme descrito anteriormente.

Foram ajustados os modelos de Brody, von Bertalanffy, Richards, Logístico e Gompertz (Tabela 1). Os parâmetros dos modelos foram estimados pelo algoritmo de Gauss Newton, descrito por HARTLEY (1961), por meio do "proc NLIN" do SAS (1990).

Nas equações apresentadas na tabela 1, a maioria dos parâmetros a serem estimados possui interpretação biológica. O parâmetro 'A' é interpretado como peso assintótico, sendo este o peso médio à maturidade, livre das variações sazonais; o parâmetro 'K' é interpretado como a taxa de maturação, quanto maior esse valor, mais precoce é o animal, e vice-versa (BROW et al., 1976). O parâmetro 'm', denominado de ponto de inflexão, refere-se ao ponto em que o animal passa de uma fase de crescimento acelerado para uma fase de crescimento inibitório, em que os ganhos em peso são menores. Na função de Richards, ele é variável e assume valores fixos nas demais funções, fazendo com que essas apresentem formas definidas. Considera-se ' $m$ ' $=1$ na função de Brody; ' $m$ '=-1 na Logística; ' $m$ '=3 na de Bertalanffy; e ' $m$ ' tendendo a infinito na de Gompertz (SILVA et al., 2001). O parâmetro 'b', que é uma constante de integração sem interpretação biológica, é utilizado apenas para adequar o valor inicial do peso vivo, fazendo com que 
Tabela 1 - Equação geral de cada modelo de crescimento utilizado para análise.

\begin{tabular}{ll}
\hline Modelo & \multicolumn{1}{c}{ Equação } \\
\hline Brody (BRODY, 1945) & $\mathrm{Y}_{\mathrm{t}}=\mathrm{A}\left(1-\mathrm{Be}^{-\mathrm{kt}}\right)+\varepsilon$ \\
Von Bertalanffy (BERTALANFFY, 1957) & $\mathrm{Y}_{\mathrm{t}}=\mathrm{A}\left(1-\mathrm{Be}^{-\mathrm{kt}}\right)^{3}+\varepsilon$ \\
Richards (RICHARDS, 1959) & $\mathrm{Y}_{\mathrm{t}}=\mathrm{A}\left(1-\mathrm{Be}^{-\mathrm{kt}}\right)^{\mathrm{m}}+\varepsilon$ \\
Logístico (NELDER, 1961) & $\mathrm{Y}_{\mathrm{t}}=\mathrm{A}\left(1-\mathrm{Be}^{-\mathrm{kt}}\right)^{-\mathrm{m}}+\varepsilon$ \\
Gompertz (LAIRD, 1965) & $\mathrm{Y}_{\mathrm{t}}=\mathrm{Ae}^{-\mathrm{Be}(-\mathrm{kt})}+\varepsilon$ \\
\hline
\end{tabular}

' $\mathrm{A}$ ' = peso assintótico ou peso médio à maturidade; ' $\mathrm{B}$ ' = constante de integração sem interpretação biológica; ' $\mathrm{k}$ ' = taxa de crescimento; e ' $\mathrm{m}$ ' = ponto de inflexão.

a curva passe pela origem, quando $y \neq 0$ e, ou $t \neq 0$. Após obtenção dos parâmetros das curvas, foram estimadas as correlações entre esses parâmetros, utilizando o "proc CORR" do SAS (1990).

Os critérios utilizados para escolha do modelo de melhor ajuste da curva de crescimento foram o coeficiente de determinação $\left(\mathrm{R}^{2}\right)$, calculado pela função 1 - (SQR/SQTc), sendo SQR a soma de quadrados do resíduo e SQTc a soma de quadrados total corrigida pela média, o desvio padrão assintótico (DPA), calculado a partir da raiz quadrada do quadrado médio do resíduo de cada modelo, e o desvio médio absoluto dos resíduos (DMA), conforme proposto por SARMENTO (2006), calculado como a seguir:

$$
D M A=\frac{\sum_{i=i}^{n}|Y i-\hat{Y} i|}{n}
$$

Para a escolha do melhor modelo, foi utilizado o índice assintótico (IA), descrito por RATKOWSKY (1990), combinando os critérios DPA, $\mathrm{DMAe}^{2}$, atribuindo valor 100 para a maior estimativa de cada critério, sendo os demais ponderados em relação a este, assim: $\mathrm{IA}=(\mathrm{DPA}+\mathrm{DMA})-\mathrm{R}^{2}$. Quanto menor o índice, melhor o ajuste do modelo.

\section{RESULTADOS E DISCUSSÃO}

As médias e os desvios padrão dos pesos, em cada uma das sete idades, são encontrados na tabela 2, na qual foram observados, para ambos os sexos, valores inferiores, a partir do $21^{\circ}$ dia de idade, aos encontrados por MAZUCHELI (2011).

Dos cinco modelos de crescimento utilizados, apenas o modelo de Richards não apresentou convergência, tanto para machos como para fêmeas (Tabela 3). Verificou-se um bom ajustamento dos modelos, tanto para machos como para fêmeas (Figura 1).

O parâmetro 'A', ou peso assintótico, interpretado como peso à maturidade, apresentou maior estimativa (Tabela 3) para o modelo de von Bertalanffy, tanto para machos $(410,9)$, como para fêmeas $(436,6)$, superestimando o peso observado aos 42 dias de idade (Tabela 2), superestimação esta também observada em frangos e frangas (FREITAS et al., 1984). Observou-se também que o peso assintótico para fêmeas, em todos os modelos, foi superior ao dos machos, o que é justificado devido ao desenvolvimento do aparelho reprodutivo e maior deposição de gordura

Tabela 2 - Média e desvio padrão dos pesos corporais (g), ao nascimento, aos 7, 14, 21, 28,35 e 42 dias de idade, de machos e fêmeas de codornas de corte.

\begin{tabular}{|c|c|c|c|c|}
\hline \multirow{2}{*}{ Idade (dia) } & \multicolumn{2}{|c|}{ Peso corporal para machos $(\mathrm{g})$} & \multicolumn{2}{|c|}{--- Peso corporal para fêmeas (g) ------- } \\
\hline & Média & Desvio padrão & Média & Desvio padrão \\
\hline 1 & 8,914 & 0,898 & 8,939 & 0,927 \\
\hline 7 & 26,035 & 5,741 & 26,265 & 5,667 \\
\hline 14 & 60,807 & 13,348 & 61,338 & 14,048 \\
\hline 21 & 106,661 & 22,159 & 108,826 & 24,545 \\
\hline 28 & 158,784 & 29,779 & 163,769 & 32,859 \\
\hline 35 & 205,478 & 30,542 & 213,976 & 32,923 \\
\hline 42 & 239,101 & 28,711 & 248,833 & 34,251 \\
\hline
\end{tabular}


Tabela 3 - Estimativa dos parâmetros das curvas de crescimento para codornas de corte da linha LF1.

\begin{tabular}{|c|c|c|c|c|c|c|c|c|c|}
\hline \multirow{2}{*}{ Modelo } & \multirow{2}{*}{ Sexo } & \multicolumn{4}{|c|}{-------------------------- Parâmetro -------------------------- } & \multirow{2}{*}{ DMA (\%) } & \multirow{2}{*}{ DPA $(\%)$} & \multirow{2}{*}{$\mathrm{R}^{2}(\%)$} & \multirow{2}{*}{ IA } \\
\hline & & A & $\mathrm{B}$ & $\mathrm{k}$ & $\mathrm{m}$ & & & & \\
\hline \multirow{2}{*}{ Brody } & Macho & 264,8 & 19,9716 & 0,1223 & - & 100,00 & 90,31 & 97,58 & 92,73 \\
\hline & Fêmea & 276,6 & 20,9912 & 0,1229 & - & 87,83 & 99,92 & 97,22 & 90,53 \\
\hline \multirow{2}{*}{ Bertalanffy } & Macho & 410,9 & 0,7887 & 0,0377 & - & 91,01 & 90,12 & 97,58 & 83,55 \\
\hline & Fêmea & 436,6 & 0,7973 & 0,0372 & - & 100,00 & 100,00 & 97,21 & 102,79 \\
\hline \multirow{2}{*}{ Gompertz } & Macho & 331,5 & 3,8975 & 0,0594 & - & 34,56 & 89,62 & 97,61 & 26,57 \\
\hline & Fêmea & 349,0 & 3,9747 & 0,0592 & - & 43,47 & 99,45 & 97,24 & 45,68 \\
\hline \multirow{2}{*}{ Logístico } & Macho & 308,7 & - & 0,0716 & 5,2478 & 26,77 & 100,00 & 97,61 & 29,16 \\
\hline & Fêmea & 324,5 & - & 0,0713 & 5,3483 & 33,28 & 88,99 & 97,25 & 25,02 \\
\hline
\end{tabular}

' $\mathrm{A}$ ' = peso assintótico ou peso médio à maturidade; ' $\mathrm{B}$ ' = constante de integração sem interpretação biológica; ' $\mathrm{k}$ ' = taxa de crescimento; $\mathrm{e}$ ' $\mathrm{m}$ ' = ponto de inflexão.

DMA - Desvio médio absoluto; DPA - Desvio Padrão Assintótico; R² - Coeficiente de Determinação; IA - Índice Assintótico.

nas fêmeas (RAJINI \& NARAHARI, 1998). Esse resultado nos mostra que tanto os machos quanto as fềmeas apresentaram peso elevado à maturidade em idade precoce, o que nos leva a inferir que essas codornas passaram por uma intensa seleção, levando em consideração o peso corporal.

Para o parâmetro ' $\mathrm{k}$ ', que representa a taxa de maturidade, ou a velocidade de crescimento para se atingir o peso assintótico, as menores estimativas foram obtidas para o modelo de von Bertalanffy, resultado este justificado pelo fato de os parâmetros ' $\mathrm{A}$ ' e ' $\mathrm{k}$ ' serem correlacionados negativamente (GARNERO et al., 2005). As maiores estimativas de ' $\mathrm{k}$ ' foram observadas para o modelo de Brody, em ambos os sexos $(0,1223$ e 0,1229 para machos e fêmeas, respectivamente).

A velocidade de crescimento é de suma importância em programas de melhoramento genético, pois, quanto mais rápido a codorna cresce, menor o intervalo de tempo para se atingir a idade adulta e, com isso, pode-se reduzir gastos com a alimentação, diminuir os intervalos de geração, e consequentemente, aumentará o ganho genético a cada geração.

Considerando os critérios para qualidade de ajuste do modelo, observa-se que o $\mathrm{R}^{2}$ não seria um bom indicador para escolha dos modelos, pois todos apresentaram valores altos e semelhantes. Em relação ao desvio padrão assintótico (DPA), também se observa uma pequena variação entre os modelos, sendo os maiores valores observados nos modelos Logístico (machos) e de von Bertalanffy (fêmeas), e os menores valores nos modelos de Gompertz (machos) e Logístico (fêmeas).

Ao analisar o desvio médio absoluto (DMA), observou-se maior valor para o modelo de von Bertalanffy para fêmeas (100\%) e o de Brody para machos $(100 \%)$, indicando que esses modelos não seriam adequados para descrever a curva de crescimento de machos e fêmeas de codornas da linha LF1. Os menores valores de DMA, tanto para machos $(26,77 \%)$ como para fêmeas $(34,56 \%)$, foram observados no modelo Logístico, indicando, segundo o DMA, ser este o melhor modelo para descrever o padrão de crescimento de codornas de corte da linha em estudo.

O menor valor para o índice assintótico (IA) foi observado para o modelo de Gompertz em codornas machos $(26,57)$ e para o Logístico em fêmeas $(25,02)$, indicando serem estes os modelos que mais se ajustaram aos dados. Considerando que o IA é um critério que combina ${ }^{2}$, DPA e DMA, e, portanto, em princípio, é considerado um critério mais "completo", sendo este o recomendado para escolha de modelos (ARAÚJO, 2009).

As correlações fenotípicas dos parâmetros foram estimadas (Tabela 4), evidenciando correlação negativa de alta magnitude entre os parâmetros ' $\mathrm{A}$ ' e ' $\mathrm{k}$ ', tanto para machos como para fêmeas, em todos os modelos avaliados, indicando que animais que atingem a maturidade mais pesados tendem a apresentar menor taxa de crescimento. Resultado similar ao obtido por SARMENTO, (2006).

A correlação positiva entre ' $m$ ' e ' $k$ ', verificada para machos e fêmeas em todos os modelos 


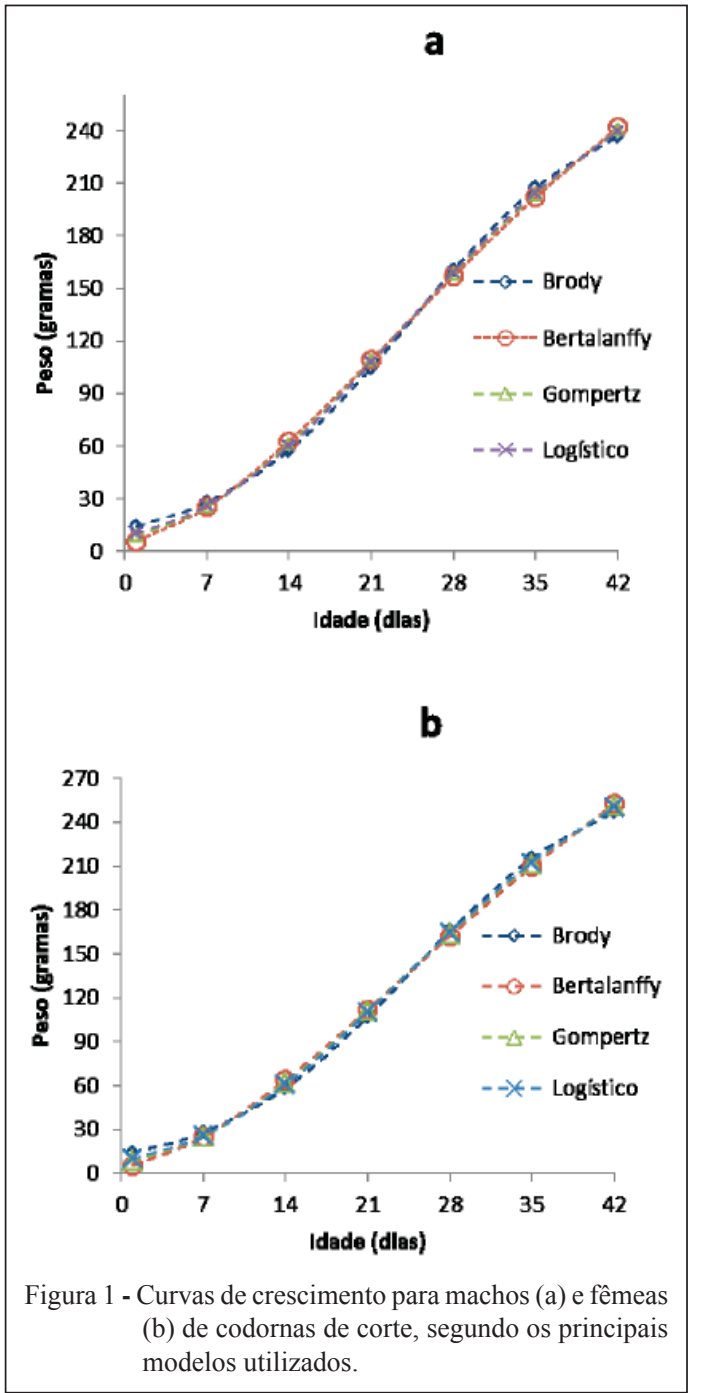

(Tabela 4) indica que quanto maior a inclinação da curvatura das funções, maior será a precocidade dos animais (THOLON \& QUEIROZ, 2009).

\section{CONCLUSÃO}

Recomenda-se a utilização do modelo de Gompertz para machos e do modelo Logístico para

Tabela 4 - Correlação fenotípica entre os parâmetros das curvas de crescimento para machos (acima da diagonal) e fêmeas (abaixo da diagonal).

\begin{tabular}{|c|c|c|c|c|c|c|c|c|c|c|c|c|}
\hline \multirow{2}{*}{ Parâmetro } & \multicolumn{6}{|c|}{ 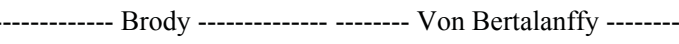 } & \multicolumn{3}{|c|}{---------- Gompertz ----------- } & \multicolumn{3}{|c|}{---------- Logístico ---------. } \\
\hline & A & $\mathrm{B}$ & $\mathrm{k}$ & A & $\mathrm{B}$ & $\mathrm{k}$ & A & $\mathrm{B}$ & $\mathrm{k}$ & A & $\mathrm{k}$ & $\mathrm{m}$ \\
\hline A & 1 & $-0,46$ & $-0,83$ & 1 & $-0,65$ & $-0,98$ & 1 & $-0,60$ & $-0,95$ & 1 & $-0,94$ & $-0,53$ \\
\hline B & $-0,47$ & 1 & 0,85 & $-0,66$ & 1 & 0,78 & $-0,61$ & 1 & 0,80 & - & - & - \\
\hline $\mathrm{K}$ & $-0,83$ & 0,85 & 1 & $-0,98$ & 0,79 & 1 & $-0,95$ & 0,80 & 1 & $-0,94$ & 1 & 0,76 \\
\hline K & - & - & - & - & - & - & - & - & - & $-0,54$ & 0,77 & 1 \\
\hline
\end{tabular}

' $\mathrm{A}$ ' = peso assintótico ou peso médio à maturidade; ' $\mathrm{B}$ ' = constante de integração sem interpretação biológica; ' $\mathrm{k}$ ' = taxa de crescimento; $\mathrm{e}$ 'm’ = ponto de inflexão.

Ciência Rural, v.43, n.10, out, 2013. 
fêmeas, para descrever o crescimento de codornas de corte.

\section{COMITÊ DE ÉTICA E BIOSSEGURANÇA}

Protocolo sob nº 007/10

\section{REFERÊNCIAS}

ARAÚJO, R.O. Componentes de covariâncias estimados por metodologia bayesiana para parâmetros biológicos obtidos por modelos não lineares para bubalinos da raça Murrah. 2009. $73 f$. Dissertação (Mestrado em Produção Animal) - Programa de Pósgraduação em Zootecnia, Universidade Federal de Santa Maria, RS.

BERTALANFFY, L. Quantitative laws in metabolism and growth Quarterly Review of Biology, v.32, n.3, p.217-230, 1957. Disponível em: <http://www.jstor.org/pss/2815257>. Acesso em: 14 nov. 2011

BRODY, S. Bioenergetics and growth. New York: Reinhold, 1945. 1023p. Disponível em: <http://pubs.acs.org/doi/ abs/10.1021/j150446a008>. Acesso em: 16 nov. 2011.

BROW, J.E. et al. A comparison of nonlinear models for describing weight-age relationships in cattle. Journal of Animal Science, v.42, n.4, p.810-818, 1976. Disponível em: <http://jas.fass.org/ content/42/4/810.short>. Acesso em: 22 nov. 2011.

CORRÊA, G.S.S. et al. Exigências em proteína bruta para codornas de corte EV1 em crescimento. Arquivo Brasileiro de Medicina Veterinária e Zootecnia, v.59, p.1278-1286, 2007. Disponível em: <http://www.scielo.br/scielo.php?script=sci_artte xt\&pid=S0102-09352007000500027>. Acesso em: 23 nov. $\overline{2} 011$ doi: 10.1590/S0102-09352007000500027.

FREITAS, A.R. et al. Modelos de curva de crescimento em frangos de corte. Pesquisa Agropecuária Brasileira, v.19, n.9, p.10571064, 1984. Disponível em: <http://orton.catie.ac.cr/cgibin/wxis. exe $/$ ? IsisScript $=$ ACERVO.xis\&method $=$ post $\&$ formato $=2 \&$ canti $\mathrm{dad}=1 \&$ expresion $=\mathrm{mfn}=041655>$. Acesso em: $02 \mathrm{de}$ dez. 2011.

GARNERO, A. del V. et al. Parâmetros genéticos da taxa de maturação e do peso assintótico de fêmeas da raça Nelore. Arquivo Brasileiro de Medicina Veterinária e Zootecnia, v.57, n.5, p.652-662, 2005. Disponível em: <http://www.scielo.br/ scielo.php?script=sci_arttext\&pid=S0102-09352005000500011>. Acesso em: 25 nov. 2011. doi: 10.1590/S0102-09352005000500011.

GOUS, R.M. Lysine: making progress in the nutrition of broilers Poultry Science, v.77, n.1, p.111-117, 1998. Disponível em: <http:// ps.fass.org/content/77/1/111.short>. Acesso em: 14 nov. 2011.

HARTLEY, H.O. The modified Gauss-Newton method for the fitting of nonlinear regression functions by least squares. Thechnometrics, v.3, p.269-280, 1961. Disponível em: <http:// www.jstor.org/pss/1266117>. Acesso em: 16 nov. 2011.

KNIZETOVA, H. et al. Analysis of growth curves of fowl. I - Chickens. Poultry Science, v.32, p.1027-1038, 1991. Disponível em: <http://www.tandfonline.com/doi/abs/10.1 080/00071669108417427>. Acesso em: 16 nov. 2011. doi $10.1080 / 00071669108417427$.
LAIRD, A.K. Dynamics of relative growyh. Growth, v.29, p.249-263, 1965. Disponível em: <http://ukpmc.ac.uk/abstract/ $\mathrm{MED} / 5865687 / \mathrm{reload}=0 ;$ jsessionid $=\mathrm{Cf} 2 \mathrm{snUO} 21$ loCJzNpyyD eU.125>. Acesso em: 18 nov. 2011.

LAIRD, A.K.; HOWARD, A. Growth curves in imbred mice. Nature, v.213, n.5078, p.786-788, 1967. Disponível em: $<$ http:// ukpmc.ac.uk/abstract/MED/6031803>. Acesso em: 13 nov. 2011. doi: $10.1038 / 213786 \mathrm{a} 0$.

MANSOUR, H. et al. Analysis of covariance structure of repeated measurements in holstein conformation traits. Journal of Dairy Science, v.74, n.8, p.2757-2766, 1991. Disponível em: <http:// download.journals.elsevierhealth.com/pdfs/journals/00220302/ PIIS0022030291784555.pdf>. Acesso em: 15 nov. 2011

MAZUCHELI, J. et al. Modelo de crescimento de Gompertz na presença de erros normais heterocedásticos: um estudo de caso. Revista Brasileira de Biometria, v.29, n.1, p.91-101, 2011.

NELDER, J.A. The fitting of a generalization of the logistic curve. Biometrics, v.17, n.1, p.89-110, 1961. Disponível em: <http:// www.jstor.org/pss/2527498>. Acesso em: 11 nov. 2011.

OLIVEIRA, E.G. et al. Desempenho produtivo de codornas de ambos os sexos para corte alimentadas com dietas com quatro níveis protéicos. Arquivo Brasileiro de Medicina Veterinária e Zootecnia, v.7, n.2, p.75-80, 2002. Disponível em: $<$ http://ojs.c3sl. ufpr.br/ojs-2.2.4/index.php/veterinary/article/view/3984/3224>. Acesso em: 19 nov. 2011

RAJINI, R.A.; NARAHARI, D. Dietary energy and protein requeriments od growing Japanese quails in the tropics. Indian Journal of Animal Sciences, v.68, n.10, p.1082-1086, 1998.

RATKOWSKY, D.A. Handbook of nonlinear regression models. New York and Basel, Marcel Dekker, 1990. 241p.

RONDON, E.O.O. et al. Modelagem computacional para produção e pesquisa em avicultura. Revista Brasileira de Ciência Avícola, v.4, n.1, p. 199-207, 2002. Disponível em: <http://redalyc. uaemex.mx/redalyc/pdf/1797/179713976001.pdf>. Acesso em: 22: nov. 2011

SARMENTO, J.L.R. et al. Estudo da curva de crescimento de ovinos Santa Inês. Revista Brasileira de Zootecnia, v.35, p.435442, 2006. Disponível em: <http://www.scielo.br/pdf/\%0D/rbz/ v35n2/a14v35n2.pdf>. Acesso em: 27 nov. 2011.

SAS Institute. SAS/STAT ${ }^{\circledR}$ user's guide: version 6. 4.ed. Cary, NC, 1990. p.1022.

SILVA, F.F. et al. Influência de fatores genéticos e ambientais sobre as estimativas dos parâmetros das funções de crescimento em gado nelore. Ciência Agrotécnica, v.25, n.5, p.1195-1205, 2001. Disponível em: <http://www.editora.ufla.br/site/ adm/upload/ revista/25-5-2001_19.pdf>. Acesso em: 27 nov. 2011.

THOLON, P.; QUEIROZ, S.A. Modelos matemáticos utilizados para descrever curvas de crescimento em aves aplicados ao melhoramento genético animal. Ciência Rural, v.39, n.7, p.22612269,2009

VELOSO, R.C. et al. Níveis de proteína bruta e energia metabolizável para codornas de corte. Acta Scientiarum Animal Sciences, no prelo, 2012. 